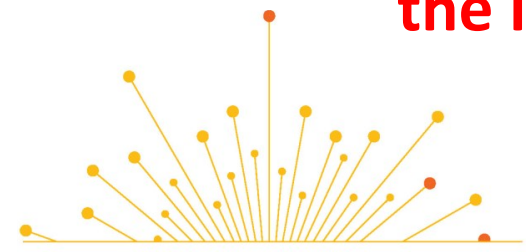

SCIENCE TABLE

COVID-19 ADVISORY FOR ONTARIO

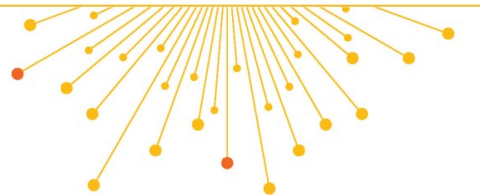

Version 1.0

Published: April 2, 2021

Citation: Pai M, Grill A, Ivers N, et al. Vaccineinduced prothrombotic immune thrombocytopenia VIPIT following AstraZeneca COVID-19 vaccination: interim guidance for healthcare professionals in the outpatient setting. Science Briefs of the Ontario COVID-19 Science Advisory Table. 2021;1(20). https:// doi.org/10.47326/ocsat.2021.02.20.1.0

Author Affiliations: The affiliations of the members of the Ontario COVID-19 Science Advisory Table can be found at https:// covid19-sciencetable.ca/.

Declarations of Interest: The declarations of interest of the members of the Ontario COVID-19 Science Advisory Table, its W ing Groups, or its partners can be found at ht covid19-sciencetable.ca/. declaration. interest of autho can be fou under ualtiona esourc at https: doi.g 10.47326/ocs 021.0 0.1.0.

Abo Us: The Onto cov 19 Scien Advi Table is a gro

and $h$ hystem lea s who evaluate and report merging dence relevant to the COVID-19 inform Ontario's response. Our mandate is to provide weekly summaries of relevant scientific evidence for the COVID-19 Health Coordination Table of the Province of Ontario, integrating information from existing scientific tables, Ontario's universities and agencies, and the best global evidence. The Science Table summarizes its findings for the Health Coordination Table and the public in Science Briefs.

The Drugs \& Biologics Clinical Practice Guidelines Working Group is a group of clinicians and scientists with recognized expertise in drugs, biologics, and clinical care. The Working Group will evaluate existing scientific data, disease epidemiology, drug availability, and implementation issues in order to develop Clinical Practice Guidelines for the treatment of COVID-19 using drugs and biologics. The Working Group reports its findings to the public and the Science Table. Its findings are also summarized in Science Briefs.

Correspondence to: Secretariat of the
SCIENCE BRIEFS-GUIDANCE FOR OUTPATIENT SETTINGS

\section{Vaccine-Induced Prothrombotic Immune Thrombocytopenia (VIPIT) Following AstraZeneca COVID-19 Vaccination}

Menaka Pai, Allan Grill, Noah Ivers, Antonina Maltsev, Katherine J. Miller, Fahad Razak, Michael Schull, Brian Schwartz, Nathan M. Stall, Robert Steiner, Sarah Wilson, Ullanda Niel, Peter Jüni, Andrew M. Morris on behalf of the Drugs \& Biologics Clinical Practice Guidelines Working Group and the Ontario COVID-19 Science Advisory Table

\section{Interim Guidance for Healthcare Professionals in the Outpatient Setting}

\section{What do we know so far?}

The AstraZeneca COVID-19 vaccine appears to be ass led th autoin pune thrombosis that mimics heparin-induced thrombo topenia $(H)$. The $t$ ited Kingdom, European Union, and Scandinav countrie ave rsport rare cas sof cerebral sinus vein thrombosis (CSVT) and thro bocytop at in patient who eived the AstraZeneca COVID-19 va The majo y of aff ted patients was far are women under tho are of 55 ea and CSVT eems to to 20 days after vaccination me like mech nism antibods that Induce massive platelet ation, $r$ ucing the latel ant d causing thrombosis. ${ }^{1}$ This phenomenon $\mathrm{mi}$ cs hepar induced rom ocytopenia (HIT) yet it does not require heparin as a trige It has $b$ an name vacc e-induced prothrombotic immune thrombocytopenia (VIPI The inci nod VIPIT appears to be between 1 in 125,000 and 1 in 1 million. $^{2}$

\section{Are cel in patients predisposed to VIPIT?}

At this time, it is not clear if certain patients are predisposed to VIPIT. The cases to date are predominantly in younger women, however these individuals may have been overrepresented in the vaccinated populations in European Union countries. ${ }^{2}$ Since VIPIT is immune-mediated, an individual with a thrombophilia, a family history of blood clots, or a personal history of arterial or venous clots would likely not be at increased of VIPIT. Accordingly, there are no new contraindications to receiving the AstraZeneca COVID-19 vaccine.

\section{What should primary care providers and patients look out for post-vaccination?}

Patients with VIPIT may present with CSVT, or with other arterial or venous clots. Some symptoms make it more likely that a patient has VIPIT: persistent and severe headache, focal neurological symptoms, seizures, or blurred or double vision (suggesting CSVT or arterial stroke); shortness of breath or chest pain (suggesting pulmonary embolism or acute coronary syndrome); abdominal pain (suggesting portal vein thrombosis); or limb swelling, redness, pallor, or coldness (suggesting deep vein thrombosis or acute limb ischemia).

\section{What should primary care providers and patients do if concerning symptoms arise?}

All patients with unusual, non-severe symptoms following vaccination should have an assessment (virtual or in-person) with their primary care provider, and a 
Ontario COVID-19 Science Advisory Table

(info@covid19-sciencetable.ca)

Copyright: 2021 Ontario COVID-19 Science Advisory Table. This is an open access document distributed under the terms of the Creative Commons Attribution License, which permits unrestricted use, distribution, and reproduction in any medium, provided that the original work is properly cited.

The views and findings expressed in this Science Brief are those of the authors and do not necessarily reflect the views of all of the members of the Ontario COVID-19 Science Advisory Table, its Working Groups, and its partners. diagnosis of VIPIT should be considered; initial investigations may be done in the primary care setting. Patients with severe symptoms should immediately present to the nearest emergency department.

Figure 1 presents a decision tree for diagnosing and ruling out VIPIT. Clinicians should ask patients about their COVID-19 vaccine history and should draw a complete blood count (CBC). VIPIT is unlikely if symptoms of blood clotting fall out of the 4-to-20-day time frame following COVID-19 vaccination OR if the platelet count is $\geq 150 \times 10^{9} /$ L. $^{3}$

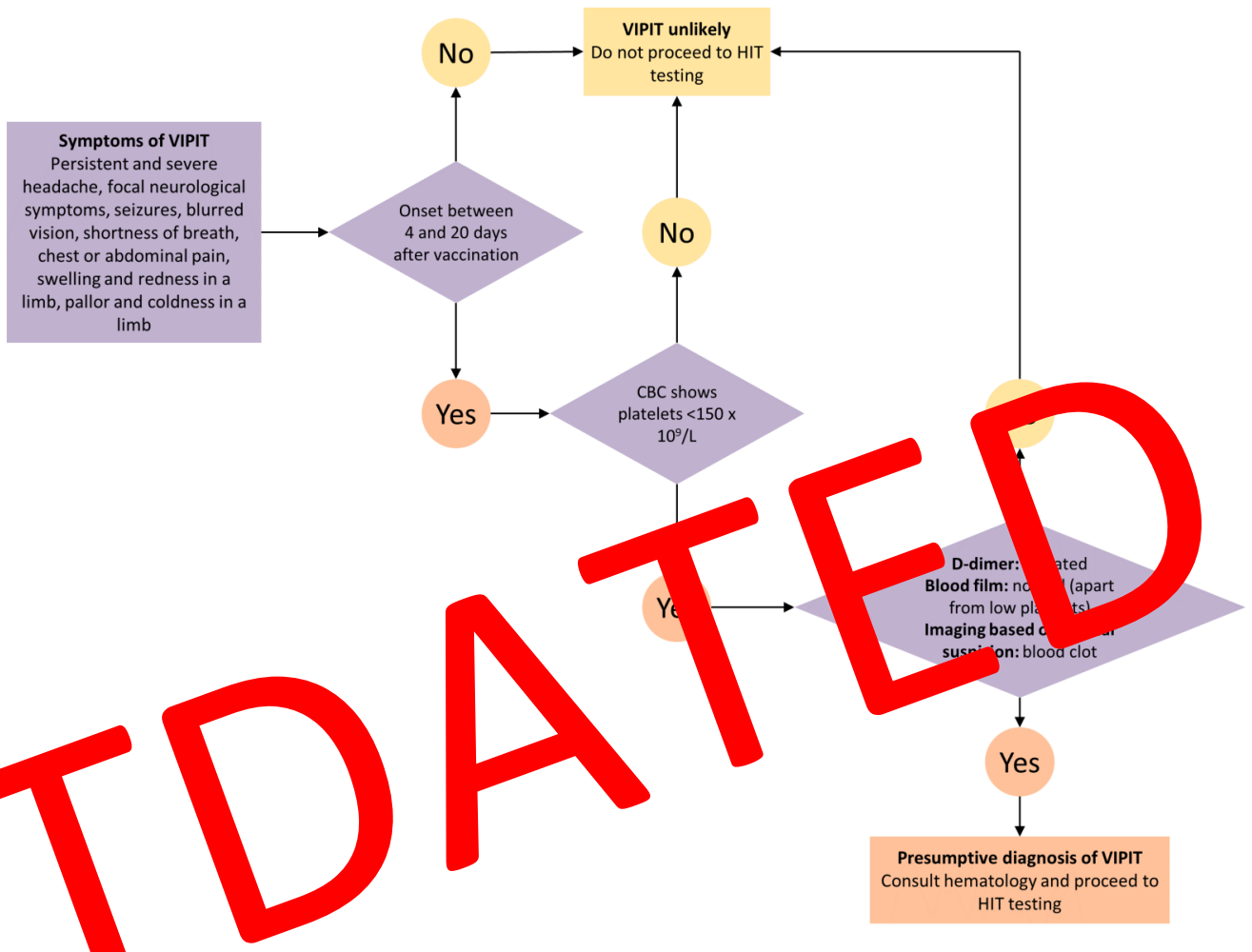

Figure 1. Lcision Tree for Diagnosing and Ruling Out VIPIT

VIPIT is more likely if symptoms of blood clotting fall in the 4-to-20-day time frame AND the platelet count is $<150 \times 10^{9} / \mathrm{L}$, and such patients should be evaluated at their nearest emergency department for suspected VIPIT. This will expedite further diagnostic workup, treatment, and urgent hematology consultation. The Box presents the treatment principles for patients with presumptive and confirmed VIPIT.

Treating Blood Clots in Patients with Presumptive or Confirmed VIPIT

1. No heparin

2. No platelet transfusions

3. First line anticoagulants: direct oral anti-Xa inhibitors (e.g., rivaroxaban, apixaban, edoxaban)

4. Consult hematology (in person, virtually, by phone)

5. IVIG $1 \mathrm{~g} / \mathrm{kg}$ daily for 2 days for severe or life-threatening blood clots

Summary Box. Treating Blood Clots in Patients with Presumptive or Confirmed VIPIT

\section{Is VIPIT a reportable event?}

All suspected adverse events following immunization (AEFI), including thrombosis, and both presumptive and confirmed VIPIT, should be reported using the provincial AEFI form and sent to the local Public Health Unit. More information on how to report AEFIs can be found on the Public Health Ontario website. Ontario conducts 
vaccine surveillance safety in collaboration with the Public Health Agency of Canada, and prompt reporting is essential to learn more about this rare but serious thrombotic phenomenon.

\section{References}

1. Updated GTH statement on vaccination with the AstraZeneca COVID-19 vaccine, as of March 22, 2021. Published March 18, 2021. Accessed March 24, 2021. https://gth-online.org/wp-content/uploads/2021/03/ GTH_Stellungnahme_AstraZeneca_3_24_2021.pdf

2. PINHO AC. COVID-19 Vaccine AstraZeneca: benefits still outweigh the risks despite possible link to rare blood clots with low platelets. European Medicines Agency. Published March 18, 2021. Accessed March 24, 2021. https:// www.ema.europa.eu/en/news/covid-19-vaccine-astrazeneca-benefits-stilloutweigh-risks-despite-possible-link-rare-blood-clots

3. Warkentin TE. Heparin-induced thrombocytopenia: pathogenesis and management. Br J Haematol. 2003;121(4):535-555. https://doi.org/10.1046/ j.1365-2141.2003.04334.x
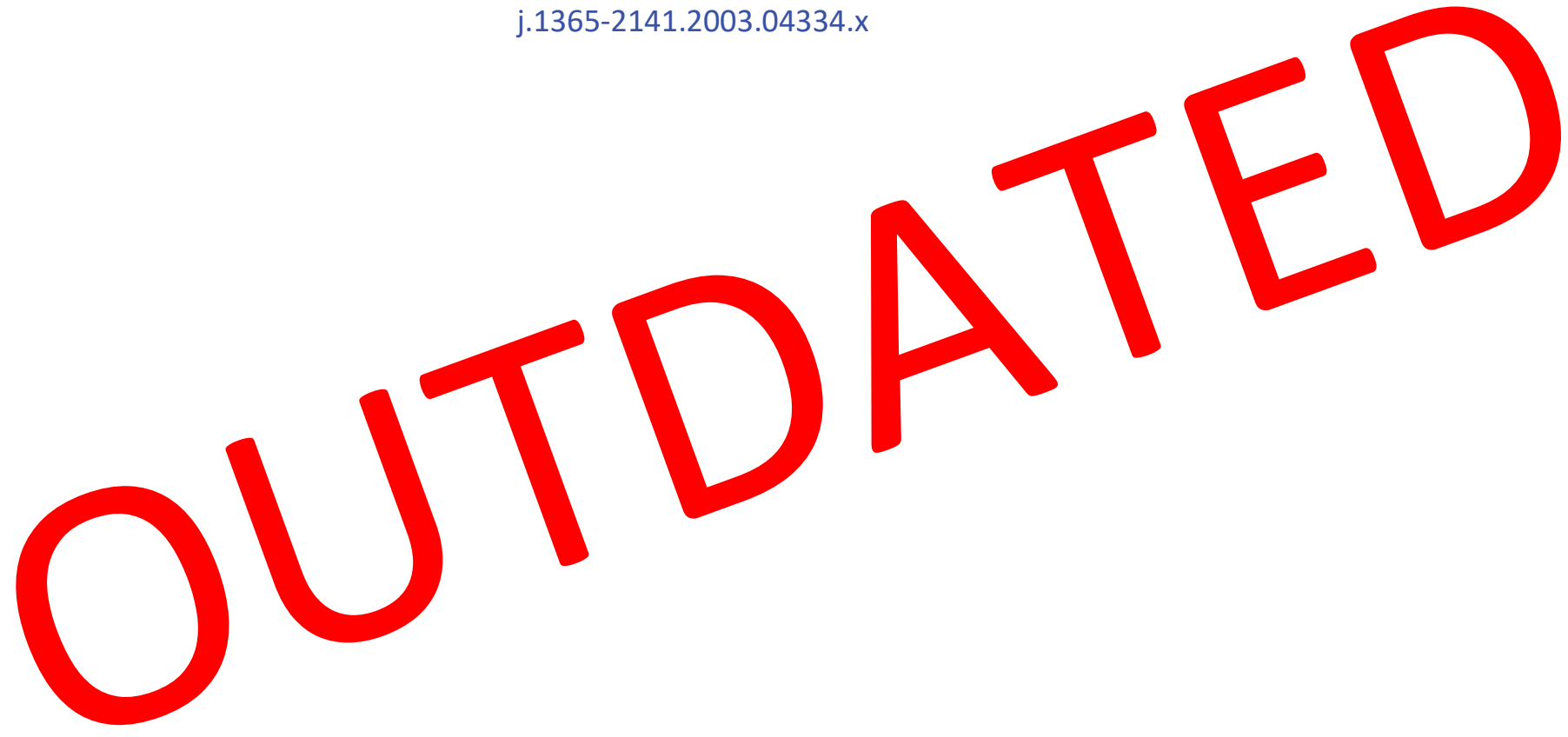